\title{
Cognitive Enhancement and Motivation Enhancement - An Empirical Comparison of Intuitive Judgments
}

\author{
Nadira Faulmüller, University of Oxford and Delft University of Technology, nadira.faulmueller@psy.ox.ac.uk \\ Thomas Douglas, University of Oxford, thomas.douglas@philosophy.ox.ac.uk \\ Felix Heise, University of Oxford, felix.heise@psy.ox.ac.uk \\ Miles Hewstone, University of Oxford, miles.hewstone@psy.ox.ac.uk
}

\begin{abstract}
SUMMARY
In an empirical study, we compared how lay people judge motivation enhancement as opposed to cognitive enhancement. We found alienation is not seen as a danger associated with either form of enhancement. Cognitive enhancement is seen as more morally wrong than motivation enhancement, and users of cognitive enhancement tend to be judged as less deserving of praise and success than users of motivation enhancement. These more negative judgments of cognitive enhancement may be driven by differences in perceived fairness rather than differences in effort exerted by the user, although lay people generally see effort as necessary to deservingness of praise and success.
\end{abstract}

\section{INTRODUCTION}

Torben Kjærsgaard (2014) notes that certain pharmaceuticals enhance both cognition and motivation and calls for greater attention to be given to the ethical issues raised specifically by their motivation-enhancing effects. In the case of cognitive enhancement, empirical research has revealed that the concerns of lay people mirror those raised in expert normative discussions (Schelle et al., 2014). This finding suggests that the views of lay people on motivation enhancement could help to inform the ethical debate, for example by suggesting ethical "hypotheses" that could be tested through normative analysis. We conducted an empirical study to examine lay people's concerns regarding motivation enhancement as compared to cognitive enhancement.

Our final sample consisted of N=188 US-American participants ( $46 \%$ female, mean age 37.5 years, mixed educational backgrounds) who completed the study online and declared that they had not previously used enhancers. Each participant read a scenario about a male student who takes enhancers while studying for exams. For $50 \%$ of participants, the scenario said that the student takes these enhancers as "smart pills; to think faster and more clearly" (cognitive enhancement scenario); for the other $50 \%$ the scenario said that the student takes these enhancers as "motivation pills; to be keener to study and overcome motivational problems" (motivation enhancement scenario). Except for these phrases, both scenarios were identical. After participants read their respective scenario, they answered several questions on 7-point Likert-scales (1 "completely disagree"; 7, "completely agree"). By comparing the averaged answers to both scenarios, we were able to assess how participants viewed motivation enhancement in comparison to cognitive enhancement. 


\section{ALIENATION}

As a potential problem related specifically to motivation enhancement, Kjærsgaard discusses alienation: artificially raised motivation might conceal that enhancement users do not actually enjoy what they are doing and cannot relate to the tasks they are performing. We found that lay people do not share this concern. Participants who read the motivation enhancement scenario tended to see the described enhancement user as highly interested in the content of his studies $(M=4.81)$ and as feeling close to the topic of his studies $(M=4.99)$. Similar results were observed for the cognitive enhancement case. Hence, lay people seem not to see a danger that motivation enhancement (or cognitive enhancement) goes along with alienation.

\section{EFFORT, FAIRNESS, AND DESERVINGNESS}

In philosophical work, it has been argued that the amount of effort people exert can influence the amount of praise or success they deserve. For example, Sorensen (2010) argues that, other things being equal, a person who exerts more effort to perform a morally good act is more deserving of praise than someone who exerts less. In the context of debate on moral enhancement, it has been entertained that if motivation enhancements decrease the effort expended in performing a morally good act, they might diminish the degree to which the enhanced individual deserves praise for that act (Douglas, 2014). One might hypothesise that, analogously, use of a motivation enhancer in a non-moral situation, for example to aid performance in an exam, would diminish the degree to which the enhanced individual deserves a good exam result and praise received for it. Moreover, if the effort-diminishing effect of enhancement is specific to motivation enhancement, as Kjærsgaard appears to believe, one might expect such enhancement to have a greater negative effect on deservingness than cognitive enhancement. We examined whether our participants made assessments of effort and deservingness that conform to this reasoning.

First, we looked at whether our participants generally perceived effort as a necessary condition for deservingness. In line with the reasoning outlined above, participants agreed with statements indicating that only those who work hard deserve praise and success $(M=5.04)$. No significant differences were observed on this general question between participants who read the cognitive enhancement scenario and participants who read the motivation enhancement scenario, so overall findings across both conditions are reported.

Using correlational analyses, we found a positive relation between regarding effort as necessary for deservingness and judgements on enhancement: the more an individual participant saw effort as necessary for deservingness, the more morally wrong they judged the use of (motivation or cognitive) enhancement $(r=.334, p<.001)$. Moreover, the more they saw effort as necessary to deservingness, the less deserving they deemed the enhancement user $(r=-.332, p<.001)$ and in turn, the more responsible they deemed the respective enhancing substance for this success $(r=.424$, $p<.001)$.

Correspondingly, the less participants perceived effort to be undermined by the use of enhancement (as measured with their rating of whether they think the described user puts effort into his work), the less morally wrong they judged (motivation or cognitive) enhancement use to be ( $r=-.363$, $p<.001)$. When looking at motivation enhancement in comparison to cognitive enhancement, we observed that participants did not think that either form of enhancement was systematically 
associated with the user putting less effort into his work than the other $\left(M_{\text {motivation }}=4.90\right.$, $M_{\text {cognitive }}=4.93, t=.174, p=.862$ ).

We did, however, find a slight difference in attributions of deservingness between participants who judged the motivation enhancement scenario and those who judged the cognitive enhancement scenario. Although this difference is only marginally statistically significant, participants tended to perceive the user of cognitive enhancement as less deserving of praise and success ( $M=4.55)$ than the user of motivation enhancement $(M=4.91, t=-1.71, p=.088)$. Hence, according to our participants, cognitive enhancement undermines deservingness slightly more than motivation enhancement does. What could explain this assessment, given that there were no differences in ascribed effort?

Advantages accrued through enhancement tended to be perceived as more unfair when the enhancement takes the form of "smart pills" $(M=4.73)$ as compared to "motivation pills" $(M=4.21$, $t=1.94, p=.054-a$ finding that approaches statistical significance). Correlational analyses tentatively suggest that this unfairness attribution may have driven the reduced deservingness perceived to be associated with cognitive enhancement as compared to motivation enhancement: the more individual participants deemed advantages acquired through enhancement as unfair, the less deserving they deemed the user to be $(r=-.720, p<.001)$ - and this relation between fairness and deservingness was of considerably greater statistical strength than the relationship between effort and deservingness reported above. From the perspective of normative ethics, this is unsurprising, since it is standardly thought that unfairly acquired gains are not deserved.

Moreover, we found that participants judged motivation enhancement as less morally wrong $(M=3.91)$ than cognitive enhancement ( $M=4.48, t=2.144, p=.033)$. Again, this overall judgement on wrongness - although significantly correlated with both - was more strongly statistically associated with unfairness $(r=.721, p<.001)$, than with effort $(r=.334, p<.001)$, suggesting that concerns about fairness may play a larger role in causing negative judgements about the morality of enhancement use than do concerns about effort.

However, it is also possible that the direction of explanation runs in the opposite direction: it may be that advantages accrued through cognitive enhancements were deemed to be more unfair than those acquired through motivation enhancements because they were less deserved and because taking enhancement is morally wrong. Further empirical work is needed to critically test the exact causal relationships between attributions of moral wrongness, deservingness, effort, and fairness in relation to motivation enhancement and cognitive enhancement. Our results shouldn't be seen as more than a preliminary step in such investigation, also because some of the statistical relations we found are weak.

\section{CONCLUSION}

Users of cognitive enhancement tend to be judged as acting more unfairly and as less deserving of praise and success than users of motivation enhancement. We have argued previously that being denied praise can be seen as a considerable psychological cost of enhancement (Faulmüller et al., 2013). Our results indicate that motivation enhancement might be associated with fewer such costs than cognitive enhancement. 
Ethical debate may be advanced by neuroscientific research that can disentangle pharmaceutical enhancers' motivational from their cognitive effects (cf. Maslen et al., 2014). Similarly, even though lay intuitions about enhancement can be affected by cognitive biases (Caviola et al., 2014), empirical investigations of the general public's views may usefully inform ethical analysis of motivation enhancement. Our findings are a first step in this direction. In raising effort-based concerns as a particular worry about motivation enhancement, Kjærsgaard implicitly suggests that these concerns are more serious in relation to motivation enhancement than cognitive enhancement. This view does not appear to be shared by our participants, perhaps indicating that it might merit scrutiny in subsequent ethical discussion. Another promising line of inquiry might be to consider whether, as our participants appeared to believe, considerations of fairness bear differently on cognitive and motivation enhancement.

\section{Manuscript ID: UABN-2014-0065}

\section{Word count: 1.500}




\section{REFERENCES}

Caviola, Lucius, Adriano Mannino, Julian Savulescu, and Nadira Faulmüller. 2014. Cognitive biases can affect moral intuitions about cognitive enhancement. Frontiers in Systems Neuroscience 8: 195. doi: 10.3389/fnsys.2014.00195.

Douglas, Thomas. 2014. Enhancing Moral Conformity and Enhancing Moral Worth. Neuroethics 7: 75-91. doi: 10.1007/s12152-013-9183-y

Faulmüller, Nadira, Hannah Maslen, and Filippo Santoni de Sio. 2013. The indirect psychological costs of cognitive enhancement. The American Journal of Bioethics 13: 45-47. doi:

$10.1080 / 15265161.2013 .794880$

Kjærsgaard, Torben. 2014. Enhancing motivation by use of prescription stimulants: The ethics of motivation enhancement. AJOB Neuroscience XX:XX.

Maslen, Hannah, Nadira Faulmüller, and Julian Savulescu. 2014. Pharmacological cognitive enhancement - how neuroscientific research could advance ethical debate. Frontiers in Systems Neuroscience 8:107. doi:10.3389/fnsys.2014.00107

Schelle, Kimberly J., Nadira Faulmüller, Lucius Caviola, and Miles Hewstone. 2014. Attitudes towards pharmacological cognitive enhancement - a review. Frontiers in Systems Neuroscience 8:53.

doi:10.3389/fnsys.2014.00053

Sorensen, Kelly. 2010. Effort and Moral Worth. Ethical Theory \& Moral Practice 13(1): 89-109. doi: 10.1007/s10677-009-9159-5 